\title{
Bootstrap current and neoclassical transport in quasi-isodynamic stellarators
}

\author{
P. Helander and J. Nührenberg \\ Max-Planck-Institut für Plasmaphysik, 17491 Greifswald, Germany
}

It is shown that the problem of neoclassical transport at low collisionality in a perfectly quasi-isodynamic (or omnigenous) stellarator can be reduced to the corresponding problem in a tokamak. In fact, the distribution function consists of two parts: one that can be calculated exactly (for any collision operator) and does not carry any net parallel current, and one that is proportional to the total toroidal current and is determined by an equation that is identical to that solved in tokamak theory. Results from the latter can therefore be carried over to the corresponding stellarator situation. Specifically, if the total toroidal current enclosed by a flux surface vanishes, then the net bootstrap current on that surface also vanishes. It is therefore consistent to optimise a stellarator in such a way that the bootstrap current and neoclassical transport are simultaneously minimised.

PACS numbers. 52.55.Hc, 52.25.Fi 


\section{Introduction}

An important line of stellarator optimisation is the quest for quasi-isodynamic configurations [1]. In a quasi-isodynamic magnetic field the cross-field drift vanishes on a time average, i.e., the field is omnigenous [2], and the trapped particles precess poloidally around the torus rather than toroidally (as in tokamak) or helically. The largest stellarator under construction, Wendelstein $7-\mathrm{X}$, is nearly quasi-isodynamic (particularly when the plasma pressure is high), and further optimisation studies have shown that quasi-isodynamicity can be achieved to a very high degree of accuracy [3].

The other main line of stellarator optimisation is the search for quasi-symmetric configurations $[4,5,6]$. Such magnetic fields are symmetric in Boozer coordinates [7], and have neoclassical properties that are identical to those in a tokamak [8]. Under a suitable transformation of variables, the drift kinetic equation is isomorphic to that in an axisymmetric magnetic field. The computation of neoclassical transport, bootstrap current, current-drive efficiency, zonal-flow damping etc can therefore be reduced to the corresponding calculations in a tokamak field.

In the present paper, we study the neoclassical properties of quasi-isodynamic plasmas in the long-mean-free-path regime. It was argued in Ref. [3] that the bootstrap current should vanish in this limit if the total current also vanishes and if the collision operator is approximated by its pitch-angle-scattering component. This is valuable since an important element of quasi-isodynamic stellarator optimisation is to reduce parallel currents as much as possible, in order to make the magnetic field structure insensitive to plasma pressure. Here we show that the bootstrap current in a quasiisodynamic field actually vanishes exactly for any collision operator at long mean-free path, if the net toroidal current vanishes. In fact, it turns out that the distribution function (and therefore all neoclassical transport coefficients determining cross-field fluxes, bootstrap current etc) consists of two terms. The first one is independent of the collision operator and can be calculated exactly. The second term is identical to that in a corresponding tokamak, but is multiplied by a coefficient proportional to the net toroidal current and therefore vanishes in a current-free stellarator.

In the next section we review some properties of omnigenous magnetic fields that we need for solving the kinetic equation in the following section. Our conclusions are summarised in the final section. 


\section{Quasi-isodynamic magnetic fields}

An omnigenous magnetic field is defined as a field where no particles drift off flux surfaces on a time average [2]. Thus, the radial drift velocity should vanish on an orbit average. In particular, this must hold for trapped particles, whose turning points thus stay on the same flux surface as they bounce back and forth. In general, they undergo precession on the surface, however, and there are three topological possibilities for how this can occur: the precession must be in the poloidal, toroidal, or a helical direction. As already mentioned, a quasi-isodynamic magnetic field is defined as an omnigenous field with poloidal trapped-particle precession.

Omnigenous magnetic fields have two remarkable properties that we shall use [9]. The first one is that the mimimum and maximum values of the field strength are the same for each field line on the same flux surface. Thus, if we write the field as $\mathbf{B}=\nabla \psi \times \nabla \alpha$, where $\psi$ is the toroidal flux and $\alpha=\theta-\iota(\psi) \varphi$, with $\theta$ and $\varphi$ poloidal and toroidal angles, then

$$
\frac{\partial B_{\max }}{\partial \alpha}=\frac{\partial B_{\min }}{\partial \alpha}=0
$$

where $B_{\max }(\psi, \alpha)$ and $B_{\min }(\psi, \alpha)$ are the maximum and minimum magnetic field strength on the field line labelled by $(\psi, \alpha)$. In a quasi-isodynamic field, the curves $B=B_{\max }$ and $B=B_{\min }$ form poloidally closed contours on each flux surface. For simplicity, we shall assume that the magnetic field only has a single maximum and minimum in each period of the stellarator. The generalisation to the case of several maxima and minima is trivial. The second property of omnigenous fields that we shall use is the fact that line integrals along the magnetic field of the kind

$$
\int_{l_{-}}^{l_{+}} f(\psi, B) d l
$$

are independent of field line, if the integration is taken between points $l_{-}(\psi, \alpha, B)$ and $l_{+}(\psi, \alpha, B)$ with equal field strength $B$ on either side of the minimum,

$$
l_{-}(\psi, \alpha, B)<l_{-}\left(\psi, \alpha, B_{\min }\right)=l_{+}\left(\psi, \alpha, B_{\min }\right)<l_{+}(\psi, \alpha, B),
$$

see Fig. 1. This result was proved by Cary and Shasharina in the appendix of Ref. [9], and thus means that

$$
\frac{\partial}{\partial \alpha} \int_{l_{-}(\psi, \alpha, B)}^{l_{+}(\psi, \alpha, B)} f(\psi, B) d l=0
$$




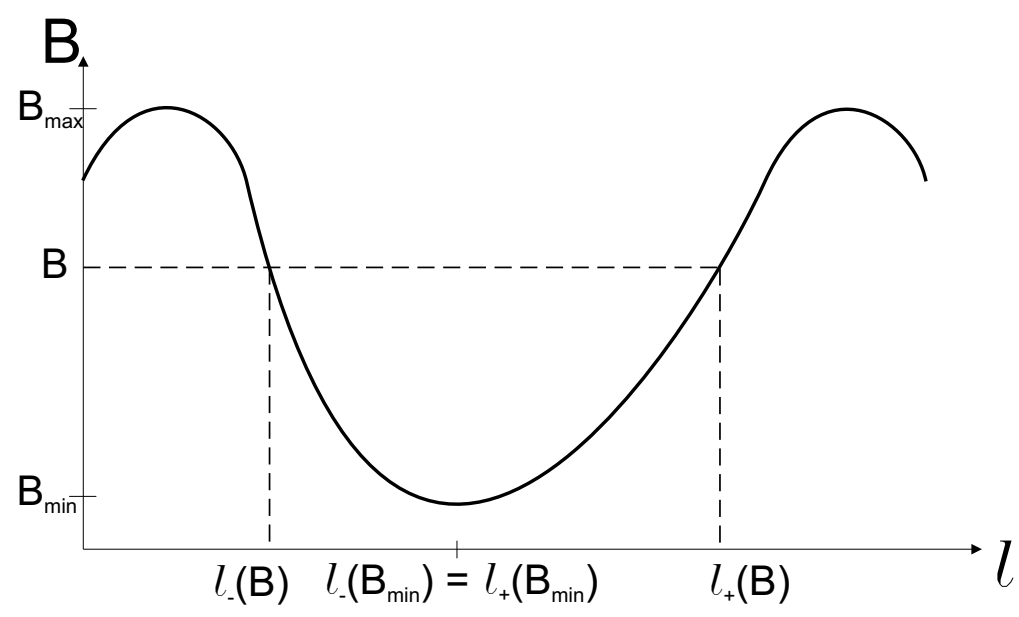

Figure 1: Magnetic field strength versus arc length.

for arbitrary $f(\psi, B)$.

It is useful to use $(\psi, \alpha, B)$ as independent coordinates within each period of the stellarator. Of course, each $B$ then corresponds to two points, $l_{-}$and $l_{+}$, which we shall refer to as the two branches of the field. The Jacobian is

$$
(\nabla \psi \times \nabla \alpha) \cdot \nabla B=\mathbf{B} \cdot \nabla B
$$

and for later use we also note that

$$
\left(\frac{\partial \mathbf{r}}{\partial \alpha}\right)_{\psi, B}=-\frac{\nabla \psi \times \nabla B}{\mathbf{B} \cdot \nabla B}
$$

When taken once around the torus poloidally, the line integral of the magnetic field is equal to $\mu_{0}$ times the enclosed toroidal current,

$$
\oint \mathbf{B} \cdot d \mathbf{r}=\oint \mathbf{B} \cdot \frac{\partial \mathbf{r}}{\partial \alpha} d \alpha=\mu_{0} J(\psi) .
$$

and it follows that there must exist a function $h(\psi, \alpha, B)$ such that

$$
\mathbf{B} \cdot \frac{\partial \mathbf{r}}{\partial \alpha}=\frac{\mu_{0} J(\psi)}{2 \pi}+\frac{\partial h}{\partial \alpha} .
$$

It is straightforward to show that the function $h$ can be chosen not to depend on the branch,

$$
\left.h(\psi, \alpha, B)\right|_{l_{-}}=\left.h(\psi, \alpha, B)\right|_{l_{+}} .
$$


To this end, consider a line integral of $\mathbf{B}$ along a closed curve, lying on a flux surface, defined by two lines of constant $\alpha$ and two lines of constant $B$ on either side of $B_{\min }$, as in Fig. 2. Let the two former curves be denoted by $C_{1}$ and $C_{3}$, and the two latter ones by $C_{2}$ and $C_{4}$. Since there is no enclosed current

$$
\oint_{C_{1}+C_{2}+C_{3}+C_{4}} \mathbf{B} \cdot d \mathbf{r}=0
$$

and because of Eq. (1),

$$
\int_{C_{1}} \mathbf{B} \cdot d \mathbf{r}=-\int_{C_{3}} \mathbf{B} \cdot d \mathbf{r}
$$

we have

$$
\int_{C_{2}} \mathbf{B} \cdot d \mathbf{r}=-\int_{C_{4}} \mathbf{B} \cdot d \mathbf{r}
$$

But Eq. (4) implies

$$
\int_{C_{2}} \mathbf{B} \cdot d \mathbf{r}=\frac{\mu_{0} J\left[\alpha\left(C_{3}\right)-\alpha\left(C_{1}\right)\right]}{2 \pi}+h\left[\alpha\left(C_{3}\right)\right]-h\left[\alpha\left(C_{1}\right)\right],
$$

so it is clear that $h$ can be chosen to be the same on the $C_{1}$ and $C_{3}$ branches.

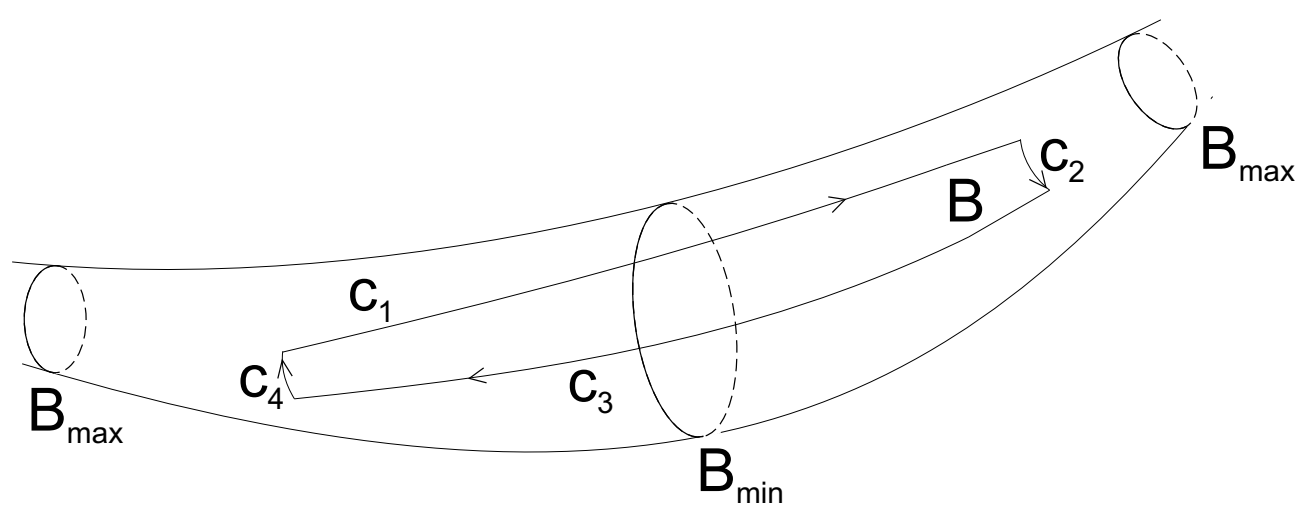

Figure 2: The curve $C_{1}+C_{2}+C_{3}+C_{4}$ lies on a flux surface and therefore does not enclose any current. The $\operatorname{arcs} C_{1}$ and $C_{3}$ coincide with field lines, while $C_{2}$ and $C_{4}$ are curves of constant field strength $|B|$ on either side of $|B|=B_{\min }$.

It is interesting to note that combining Eqs. (3) and (4) gives

$$
-\frac{(\mathbf{B} \times \nabla \psi) \cdot \nabla B}{\mathbf{B} \cdot \nabla B}=\frac{\mu_{0} J(\psi)}{2 \pi}+\frac{\partial h}{\partial \alpha} .
$$

It was shown in Ref. [10] that a magnetic field is quasisymmetric if, and only if, the left-hand side of this equation is a flux function. In the present context quasisymmetry therefore corresponds to $h=0$. 


\section{Neoclassical transport}

When expanded in the smallness of the gyroradius, the drift kinetic equation for each species $a$ becomes in first order

$$
v_{\|} \nabla_{\|} f_{a 1}+\mathbf{v}_{d} \cdot \nabla f_{a 0}+\frac{e_{a} v_{\|} \nabla_{\|} \phi_{1}}{T_{a}} f_{a 0}=C_{a}\left(f_{a 1}\right)+S,
$$

where the differentiations are carried out at constant unperturbed energy, $m_{a} v^{2} / 2+$ $e_{a} \phi_{0}(\psi)$, and normalised magnetic moment, $\lambda=v_{\perp}^{2} / v^{2} B$. The electrostatic potential is a flux function in lowest order but may vary in first order, $\phi=\phi_{0}(\psi)+\phi_{1}(\psi, \theta, \varphi)$. We have included a source term, $S$, on the right-hand side of Eq. (5) to represent contributions from neutral-beam injection, RF heating and current drive etc, but will assume that they are weak enough that the lowest-order distribution function remains Maxwellian. The transport and currents driven by $S$ then add linearly to those driven by radial gradients, which are our focus here.

Because the orbit average of the radial drift velocity vanishes by assumption, we can write

$$
\mathbf{v}_{d} \cdot \nabla f_{a 0}=-v_{\|} \nabla_{\|} F_{a}
$$

for some function $F_{a}$ that is odd in $v_{\|}$and satisfies

$$
\nabla_{\|} F_{a}=-(\mathbf{b} \times \nabla \psi) \cdot \nabla\left(\frac{v_{\|}}{\Omega_{a}}\right) \frac{\partial f_{a 0}}{\partial \psi},
$$

where $\mathbf{b}=\mathbf{B} / B$ and $\Omega_{a}=e_{a} B / m_{a}$. We can now write Eq. (5) as

$$
v_{\|} \nabla_{\|}\left(f_{a 1}-F_{a}+\frac{e_{a} \phi_{1}}{T_{a}} f_{a 0}\right)=C_{a}\left(f_{a 1}\right)
$$

and expand $g_{a}=f_{a 1}-F_{a}+\left(e_{a} \phi_{1} / T_{a}\right) f_{a 0}=g_{a 0}+g_{a 1}+\ldots$ as usual in the smallness of the collisionality. In lowest order, then, we have $\nabla_{\|} g_{a 0}=0$, and $g_{a 0}$ is determined from the next-order constraint

$$
\left\langle\frac{B}{v_{\|}} C_{a}\left(F_{a}+g_{a 0}\right)\right\rangle=0
$$

for circulating particles, $\lambda<1 / B_{\max }$, while it vanishes in the trapped region, $\lambda>$ $1 / B_{\max }$. Angular brackets denote the flux-surface average, defined as

$$
\langle\cdots\rangle=\oint d \alpha \int_{l_{-}\left(B_{\max }\right)}^{l_{+}\left(B_{\max }\right)}(\cdots) \frac{d l}{B} / \oint d \alpha \int_{l_{-}\left(B_{\max }\right)}^{l_{+}\left(B_{\max }\right)} \frac{d l}{B} .
$$


If the winding number $\iota(\psi)$ is irrational, the average in Eq. (7) is equivalent to an integral along the orbit over a large number of periods. In Eq. (7) it is understood that $\lambda$ is kept constant in $v_{\|}=\sigma v[1-\lambda B(l)]^{1 / 2}$, with $\sigma= \pm 1$.

We now solve Eq. (6),

$$
(\mathbf{b} \cdot \nabla B)\left(\frac{\partial F_{a}}{\partial B}\right)_{\psi, \alpha, v, \lambda}=-[(\mathbf{b} \times \nabla \psi) \cdot \nabla B] \frac{\partial}{\partial B}\left(\frac{v_{\|}}{\Omega_{a}}\right) \frac{\partial f_{a 0}}{\partial \psi},
$$

recalling Eqs. (2) and (3), which imply

$$
\frac{\partial F_{a}}{\partial B}=\mathbf{B} \cdot \frac{\partial \mathbf{r}}{\partial \alpha} \frac{\partial}{\partial B}\left(\frac{v_{\|}}{\Omega_{a}}\right) \frac{\partial f_{a 0}}{\partial \psi}
$$

so that

$$
F_{a}=-\frac{\partial f_{a 0}}{\partial \psi} \Delta_{a}
$$

where

$$
\Delta_{a}=-\frac{\mu_{0} J(\psi)}{2 \pi} \frac{v_{\|}}{\Omega_{a}}+\frac{\partial}{\partial \alpha} \int_{B}^{B_{\max }} h \frac{\partial}{\partial B^{\prime}}\left(\frac{v_{\|}^{\prime}}{\Omega_{a}^{\prime}}\right) d B^{\prime}, \quad \lambda<1 / B_{\max }
$$

for passing particles and

$$
\Delta_{a}=-\frac{\mu_{0} J(\psi)}{2 \pi} \frac{v_{\|}}{\Omega_{a}}+\frac{\partial}{\partial \alpha} \int_{B}^{1 / \lambda} h \frac{\partial}{\partial B^{\prime}}\left(\frac{v_{\|}^{\prime}}{\Omega_{a}^{\prime}}\right) d B^{\prime}, \quad \lambda>1 / B_{\max }
$$

for trapped ones. Here $\Omega_{a}^{\prime}=e_{a} B^{\prime} / m_{a}, v_{\|}^{\prime}=\sigma v\left(1-\lambda B^{\prime}\right)^{1 / 2}$, and we have used Eq. (4). The first term on the right of Eqs. (8) and (9) is similar to that in the corresponding tokamak calculation while the second term only arises if the parallel variation of $B$ depends on the field-line label $\alpha$. However, the contribution from this term to Eq. (7) vanishes, so that

$$
\oint d \alpha \int_{l_{-}\left(\psi, \alpha, B_{\max }\right)}^{l_{+}\left(\psi, \alpha, B_{\max }\right)} C_{a}\left(F_{a}\right) \frac{d l}{v_{\|}}=\mu_{0} J(\psi) \int_{l_{-}\left(\psi, \alpha, B_{\max }\right)}^{l_{+}\left(\psi, \alpha, B_{\max }\right)} C_{a}\left(\frac{v_{\|}}{\Omega_{a}} \frac{\partial f_{a 0}}{\partial \psi}\right) \frac{d l}{v_{\|}} .
$$

This is, in fact, the crucial step of our analysis, and follows from the fact that since $\partial / \partial \alpha$ commutes with the collision operator [11], the quantity

$$
\oint d \alpha \int_{l_{-}\left(\psi, \alpha, B_{\max }\right)}^{l_{+}\left(\psi, \alpha, B_{\max }\right)} C_{a}\left(\frac{\partial H}{\partial \alpha}\right) \frac{d l}{v_{\|}}
$$

vanishes for any function $H(\psi, \alpha, B, v, \lambda, \sigma)$ because of the Cary-Shasharina theorem (1). As a result, the constraint (7) determining $g_{a 0}$ becomes

$$
\int_{l_{-}\left(B_{\max }\right)}^{l_{+}\left(B_{\max }\right)} C_{a}\left(\frac{\mu_{0} J v_{\|}}{\Omega_{a}} \frac{\partial f_{a 0}}{\partial \psi}+g_{a 0}\right) \frac{d l}{v_{\|}}=0
$$


which is identical to that solved in neoclassical theory for tokamaks. Indeed, in the tokamak case the magnetic field is $\mathbf{B}=I\left(\psi_{p}\right) \nabla \varphi+\nabla \varphi \times \nabla \psi_{p}$, where $\psi_{p}$ is the poloidal flux, and the distribution function is determined from [12]

$$
\int C_{a}\left(-\frac{I v_{\|}}{\Omega_{a}} \frac{\partial f_{a 0}}{\partial \psi_{p}}+g_{a 0}\right) \frac{d l}{v_{\|}}=0
$$

which coincides with Eq. (10) if $\mu_{0} J$ is replaced by $-q I$, where $q=\iota^{-1}=d \psi / d \psi_{p}$. Note that $g_{a 0}$ is proportional to $J(\psi)$ and vanishes identically in a current-free stellarator.

We are now in a position to calculate the bootstrap current and cross-field transport. The parallel current carried by $f_{a 1}$,

$$
j_{a \|}=e_{a} \int v_{\|} f_{a} d^{3} v=j_{a t}+j_{a s}
$$

consists of two parts: a "tokamak part",

$$
j_{a t}=\frac{\mu_{0} J}{2 \pi B}\left(\frac{d p_{a}}{d \psi}+n_{a} e_{a} \frac{d \phi_{0}}{d \psi}\right)+e_{a} \int v_{\|} g_{a} d^{3} v,
$$

and a "stellarator part",

$$
j_{a s}=-\pi e_{a} B \sum_{\sigma} \sigma \int_{0}^{\infty} \frac{\partial f_{a 0}}{\partial \psi} v^{3} d v \int_{0}^{1 / B} d \lambda \frac{\partial}{\partial \alpha} \int_{B}^{\min \left(\lambda^{-1}, B_{\max }\right)} h \frac{\partial}{\partial B^{\prime}}\left(\frac{v_{\|}^{\prime}}{\Omega_{a}^{\prime}}\right) d B^{\prime},
$$

due to the non-equivalence of different field lines on the same flux surface, $\partial / \partial \alpha \neq 0$. The "stellarator part" of the bootstrap current does not contribute to the net toroidal current, which can be seen as follows. Its contribution, $J_{a s}(\psi)$, to the total parallel current inside a flux surface $\psi$ can be calculated by evaluating the current crossing a surface of constant $B$,

$$
J_{a s}(\psi)=\int j_{a s} \mathbf{b} \cdot d \mathbf{S}
$$

where

$$
d \mathbf{S}=\frac{\nabla B}{(\nabla \psi \times \nabla \alpha) \cdot \nabla B} d \psi d \alpha
$$

and the integral is taken over the area enclosed by the flux surface $\psi$. The result,

$$
J_{a s}(\psi)=\int_{0}^{\psi} d \psi^{\prime} \int_{0}^{2 \pi} \frac{j_{a s}}{B} d \alpha=0
$$

vanishes because of the $\alpha$-derivative in Eq. (11). (A physical interpretation of this result is provided in the concluding section.) The net toroidal current inside the flux surface $\psi$ is therefore given by

$$
J(\psi)=\sum_{a} \int\left(j_{a t} \mathbf{b}+\mathbf{j}_{a \perp}\right) \cdot d \mathbf{S}+J_{\mathrm{RF}}(\psi)+J_{\mathrm{NBI}}(\psi),
$$


where the two last terms represent any RF- and neutral-beam driven currents, and where the perpendicular current is

$$
\int \mathbf{j}_{a \perp} \cdot d \mathbf{S}=\int \frac{\mathbf{b} \times\left(\nabla p_{a}+n_{a} e_{a} \nabla \phi_{0}\right)}{B} \cdot d \mathbf{S}
$$

Using

$$
\frac{\mathbf{b} \times \nabla \psi}{B} \cdot d \mathbf{S}=\frac{\nabla \psi \times \nabla B}{\mathbf{B} \cdot \nabla B} \cdot \frac{\mathbf{B}}{B^{2}} d \psi d \alpha=-\mathbf{B} \cdot\left(\frac{\partial \mathbf{r}}{\partial \alpha}\right) \frac{d \psi d \alpha}{B^{2}}
$$

we find

$$
\int \mathbf{j}_{a \perp} \cdot d \mathbf{S}=-\frac{\mu_{0}}{B^{2}} \int_{0}^{\psi}\left(\frac{d p_{a}}{d \psi^{\prime}}+n_{a} e_{a} \frac{d \phi_{0}}{d \psi^{\prime}}\right) J\left(\psi^{\prime}\right) d \psi^{\prime} .
$$

The "tokamak part" of the bootstrap current on the surface $\psi$ is proportional to $J(\psi)$ and therefore vanishes if the net current inside this surface is zero. The same holds for the diamagnetic current according to Eq. (12), and we conclude that there is no net pressure-driven current on a flux surface inside which the total toroidal current vanishes.

The neoclassical transport can be calculated entirely as in a tokamak. The neoclassical radial particle flux is

$$
\left\langle\boldsymbol{\Gamma}_{a} \cdot \nabla \psi\right\rangle=\left\langle\int f_{a 1} \mathbf{v}_{d} \cdot \nabla \psi d^{3} v\right\rangle=-\sum_{\sigma}\left\langle B \int_{0}^{\infty} \pi v^{3} d v \int_{0}^{1 / B} f_{a 1} \nabla_{\|} F_{a} d \lambda\right\rangle .
$$

By using the fact that $\langle\mathbf{B} \cdot \nabla f\rangle=0$ for any function $f$ and

$$
\left\langle B \int_{0}^{1 / B} f_{a 1} \nabla_{\|} F_{a} d \lambda\right\rangle=-\left\langle B \int_{0}^{1 / B} F_{a} \nabla_{\|} f_{a 1} d \lambda\right\rangle,
$$

we can express the particle flux as

$$
\left\langle\boldsymbol{\Gamma}_{a} \cdot \nabla \psi\right\rangle=\left\langle\int F_{a} v_{\|} \nabla_{\|} f_{a 1} d^{3} v\right\rangle=\left\langle\int F_{a} C_{a}\left(F_{a}+g_{a}\right) d^{3} v\right\rangle,
$$

and the heat flux analogously as

$$
\left\langle\mathbf{q}_{a} \cdot \nabla \psi\right\rangle=\left\langle\int\left(\frac{m_{a} v^{2}}{2}-\frac{5 T_{a}}{2}\right) F_{a} C_{a}\left(F_{a}+g_{a}\right) d^{3} v\right\rangle .
$$

\section{Conclusions}

In a quasi-isodynamic stellarator, the first-order distribution function of each species in the limit of long mean-free path consists of two terms: a tokamak-like term and a term specific to stellarators, i.e.,

$$
f_{a}=\left(1+\frac{e_{a} \phi_{1}}{T_{a}}\right) f_{a 0}+f_{a t}+f_{a s}
$$


with

$$
\begin{gathered}
f_{a t}=g_{a}+\frac{\mu_{0} J(\psi) v_{\|}}{2 \pi \Omega_{a}} \frac{\partial f_{a 0}}{\partial \psi} \\
f_{a s}=-\frac{\partial f_{a 0}}{\partial \psi} \frac{\partial}{\partial \alpha} \int_{B}^{\min \left(B_{\max }, \lambda^{-1}\right)} h \frac{\partial}{\partial B^{\prime}}\left(\frac{v_{\|}^{\prime}}{\Omega_{a}^{\prime}}\right) d B^{\prime}
\end{gathered}
$$

The term $f_{a t}$ is proportional to the enclosed toroidal current $J(\psi)$ and is determined by a kinetic constraint (10) that is identical to that solved in neoclassical tokamak theory. This term therefore coincides with the distribution function in a tokamak with a similar parallel variation of $B$. The other term, $f_{a s}$, is due to the fact that this variation is in general different for different field lines in a stellarator. This term is however independent of the collision operator and does not carry any net bootstrap current. It follows that if the total toroidal current enclosed by a certain flux surface vanishes, then the bootstrap current on that surface also vanishes. In fact, the current carried by each species then vanishes separately on a flux-surface average. On the other hand, if the enclosed current does not vanish, then the bootstrap current can be calculated exactly as in a tokamak.

Although the bootstrap current in a perfectly quasi-isodynamic stellarator coincides with that in a tokamak, the cross-field neoclassical transport does not. This transport is tokamak-like in the sense that there is no " $1 / \nu$-regime" where the transport is inversely proportional to the collision frequency, but the stellarator-specific part of the distribution function does contribute transports. Like the tokamak-part, it does so at a rate proportional to the collision frequency. The particle and heat fluxes are given by Eqs. (13) and (14), respectively. A difference to the tokamak is that the particle flux is not only driven by collisions between different species, but also by like-particle collisions [10], even if the magnetic field is perfectly omnigenous. In practice it is difficult to achieve perfect omnigenity, so remnants of a " $1 / \nu$-regime" remains in the radial transport. The transport rates calculated here represent a lower bound, which can be approached but never quite achieved in practice. The bootstrap current, however, has no $1 / \nu$-enhancement and should be more accurately described by our results.

The fact that the "stellarator contribution" to the bootstrap current vanishes can be understood in terms of particle orbits. In the tokamak, the bootstrap current arises because of a diamagnetic effect associated with the banana orbits. Particles have positive parallel velocity (relative to the toroidal current) on the outer leg of banana orbits and 
negative $v_{\|}$on the inner leg. This is seen from the first term on the right in Eqs. (8) and (9), which represents the radial displacement (in terms of $\psi$ ) of passing and trapped particles, respectively. If the enclosed current vanishes, then the these terms vanish and only the second terms in these equations remain. This is the radial displacement due to the non-equivalence of different field lines on the same flux surface. Because of the $\alpha$-derivative, this part of the displacement changes sign over the flux surface. This means that the parallel velocity is no longer preferentially positive on the outer leg of the particle orbits, and there is no net drive for a bootstap current. Geometrically, this can be understood from the fact that, unlike in a tokamak, the trapped orbits do not reside only on the outboard side of the torus, but are distributed poloidally around the flux surface. An explicit example can be seen in Fig. 3c of Ref. [13].

The practical importance of our results is the realisation that two of the optimisation criteria for stellarators of the Wendelstein 7-X type, namely, the simulataneous minimisation of parallel currents and neoclassical transport, are mutually consistent. In Wendelstein $7-\mathrm{X}$, it is intended to use electron cyclotron current drive to cancel the residual bootstrap current $[14,15]$. This is necessary only because the neoclassical transport optimisation is not perfect. In a strictly quasi-isodynamic device, the bootstrap current and the toroidal projection of the diamagnetic current vanish exactly on a flux surface average at low collisionality.

\section{Acknowledgment}

It is a pleasure to acknowledge helpful discussions with Craig Beider, Peter Catto, Jeffrey Freidberg and Henning Maaßberg. 


\section{References}

[1] S. Gori, W. Lotz, and J. J. Nührenberg, in Theory of Fusion Plasmas (Editrice Compositori, Bologna, 1996), p. 335.

[2] L.S. Hall and B. McNamara, Phys. Fluids 18, 552 (1975).

[3] A.A. Subbotin, M.I. Mikhailov, V.D. Shafranov, M. Yu. Isaev, C. Nührenberg, J. Nührenberg, R. Zille, V.V. Nemov, S.V. Kasilov, V.N. Kaluzhnyj, and W.A. Cooper, Nucl. Fusion 46, 921 (2006),

[4] J. Nührenberg, W. Lotz, S. Gori, in Theory of Fusion Plasmas (Editrice Compositori, Bologna, 1994), p. 3.

[5] J. Nührenberg and R. Zille, Phys. Lett. A 129, 113 (1988).

[6] A.H. Boozer, Plasma Phys. Control. Fusion 37, A103 (1995).

[7] A.H. Boozer, Phys. Fluids 24, 1999 (1981).

[8] A.H. Boozer, Phys. Fluids 26, 496 (1983).

[9] J.R. Cary and S. Shasharina, Phys. Plasmas 4, 3323 (1997).

[10] P. Helander and A.N. Simakov, Phys. Rev. Lett 101, 145003 (2008).

[11] The gyro-averaged collision operator is an integro-differential operator, where the integrations and differentiations are carried out with respect to $v$ and $\xi=v_{\|} / v=$ $\sigma(1-\lambda B)^{1 / 2}$. The latter operations commute with $\partial / \partial \alpha$ but not with $\partial / \partial B$.

[12] P. Helander and D.J. Sigmar, Collisional transport in magnetized plasmas (Cambridge University Press, 2002).

[13] W.A. Cooper, M.Yu. Isaev, M.I. Mikhailov, J. Nührenberg, S. Okamura, V.D. Shafranov, A.A. Subbotin, C. Suzuki, K. Yamazaki, and R. Zille, 32nd EPS Conference on Plasma Physics (Tarragona, 2005), Vol 29C, P-2.120 (2005).

[14] H. Maassberg, W. Lotz, and J. Nührenberg, Phys. Fluids B 5, 3728 (1993).

[15] Yu. Turkin, H. Maassberg, C. D. Beidler, J. Geiger, and N. B. Marushchenko, Fusion Science and Technology 50, 387 (2006). 